\title{
Multi-color photometry of the Galactic globular cluster M 75 = NGC 6864. A new sensitive metallicity indicator and the position of the horizontal branch in UV $\star, \star \star$
}

\author{
V. Kravtsov ${ }^{1,2}$, G. Alcaíno ${ }^{3}$, G. Marconi ${ }^{4}$, and F. Alvarado ${ }^{3}$ \\ 1 Instituto de Astronomía, Universidad Católica del Norte, Avenida Angamos 0610, Antofagasta, Chile \\ e-mail: vkravtsov@ucn.cl \\ 2 Sternberg Astronomical Institute, University Avenue 13, 119899 Moscow, Russia \\ 3 Isaac Newton Institute of Chile, Ministerio de Educación de Chile, Casilla 8-9, Correo 9, Santiago, Chile \\ e-mail: inewton@terra.cl, falvarad@eso.org \\ ${ }^{4}$ ESO - European Southern Observatory, Alonso de Cordova 3107, Vitacura, Santiago, Chile \\ e-mail: gmarconi@eso.org
}

Received 9 November 2006 / Accepted 22 March 2007

\begin{abstract}
Aims. We carry out and analyze new multi-color photometry of the Galactic globular cluster (GC) M 75 in $U B V I$ and focus on the brighter sequences of the color-magnitude diagram (CMD), with particular emphasis on their location in $U$-based CMD. Specifically, we study the level both of the horizontal (HB) and red giant branches (RGB) relative to the main-sequence turnoff (TO) in the $U$ magnitude.

Methods. Along with the presented photometry of M 75, we use our collection of photometric data on GCs belonging to the metalpoor range, $[\mathrm{Fe} / \mathrm{H}]_{Z W}<-1.1$ dex, obtained from observations with different equipment, but calibrated by standard stars situated in the observed cluster fields.

Results. We confirm our earlier finding, and extend it to a larger magnitude range. We demonstrate that $\Delta U_{\mathrm{TO}}^{\mathrm{BHB}}$ expressing the difference in $U$ magnitude between the TO point and the level of the blue HB, near its red boundary, of the metal-poor GCs observed with the EMMI camera of the NTT/ESO telescope is about 0.4-0.5 mag smaller as compared to GCs observed with the 100" telescope and $1.3 \mathrm{~m}$ Warsaw telescope of the Las Campanas Observatory. At the same time, $\Delta U_{\mathrm{TO}}^{\mathrm{RGB}}$, the difference in $U$ magnitude between the TO and RGB inflection (brightest) points, does not show such an apparent dependence on the characteristics of $U$ filters used, but it depends on cluster metallicity. We have shown, for the first time, the dependence of the parameter $\Delta U_{\mathrm{TO}}^{\mathrm{RGB}}$ on $[\mathrm{Fe} / \mathrm{H}]$ and have estimated its analytical expression, by assuming a linear relation between the parameter and metallicity. Its slope, $\Delta U_{\mathrm{TO}}^{\mathrm{RGB}} / \Delta[\mathrm{Fe} / \mathrm{H}] \sim$ $1.2 \mathrm{mag} / \mathrm{dex}$, is approximately a factor of two steeper than that of the dependence of the RGB bump position in the $V$ magnitude on metallicity. The asymptotic giant branch (AGB) clump and features of the RGB luminosity function (LF) of M 75 are also discussed.
\end{abstract}

Key words. Galaxy: globular clusters: general - Galaxy: globular clusters: individual: M 75 - stars: population II

\section{Introduction}

Until recently, the southern Galactic globular cluster NGC 6864 (M 75) has been among the least studied ones. Photographic photometry in the GC by Harris (1975), reaching the cluster HB level, was the only CMD study of the globular. Recent publications include an extensive study of M 75 by Catelan et al. (2002), based on deep multi-color photometry reaching the cluster main sequence, the HST CMD of the cluster obtained by Piotto et al. (2002), as well as a study of a large number of the cluster variable stars (Corwin et al. 2003).

Using the present photometry of M 75, particularly in the $U$ bandpass, we extend our results obtained in a series of

* Based on observations with the $1.3 \mathrm{~m}$ Warsaw telescope at Las Campanas Observatory.

$\star \star$ Individual photometry measurements are only available in electronic form at the CDS via anonymous ftp to

cdsarc.u-strasbg.fr $(130.79 .128 .5)$ or via

http://cdsweb.u-strasbg.fr/cgi-bin/qcat?J/A+A/469/529 earlier studies of Galactic GCs. Carrying out photometry in GCs in the $U$ bandpass has been irregular. As a result, there is a deficit of systematic studies of $U$-based CMDs over a wide luminosity range of cluster stars, as well as over a wide metallicity range of GCs. As a rule, $U$ photometry focuses on the distribution of cluster stars along the HB in the $(U-V)$ or $(U-B)$ colors. However, deep photometry in GCs in this bandpass can be valuable for other applications as well.

Alcaíno et al. (1997b) revealed, for the first time, a surprising disagreement between the positions of the HB both in the $(U-B)-(B-V)$ two-color diagram and in the $U-(B-V)$ color-magnitude diagrams of NGC 6541 and M 79, GCs with similar blue HB (BHB) morphology and metallicity. In particular, the disagreement of the BHB position in the $U$ magnitude in the $U-(B-V)$ CMD of the two GCs was around $\Delta U \sim 0.4$ to 0.5 mag, while the slope of their RGBs was the same within the error. The most pronounced difference in the $U$ magnitude or in the $(U-B)$ color between the BHB stars of NGC 6541 and 
M 79 occurred in the color range $\Delta(B-V) \sim 0.2$ mag blue-ward of the red boundary of the BHB. The same kind of discrepancy was also found comparing the corresponding diagrams of M 79 and M 80 (Alcaíno et al. 1998b).

Later, Momany et al. (2003) reported the so-called BHB red incursion through the RGB in the $(U-B)$ color in the UV color-magnitude diagrams. The incursion is another manifestation of the same effect. Momany et al. (2003) have investigated in detail the origin of this effect and have convincingly showed that it is mainly due to the difference in transmission curves of $U$ filters available at different observatories. Specifically, transmission curves of some $U$ filters used encompass the Bulmer jump, in conformity with the standard $U B V$ photometric system, and using such filters one can realize photometric bandpasses close to the standard $U$ one. The maxima of their response curves fall between $3700-3750 \AA$ or very close to this range. In contrast to these filters, transmission curves of other $U$ filters (hereafter referred to as $U^{\prime}$ filters) are shifted blue ward of the Balmer jump, and the resulting photometric band (hereafter $U^{\prime}$ bandpass) nearly does not include this jump. The $U^{\prime}$ bandpass response curves' maxima are approximately 150-200 $\AA$ or even more blue-shifted, as compared to the location of the corresponding maxima of the $U$ bandpasses. A number of examples of the normalized transmission curves of UV filters used at different observatories are collected and presented in Momany et al. (2003).

The available information about the effect under consideration is usually expressed in the relative change of the mutual position (either in $U$-based colors or in $U$ magnitude, obtained with $U$ and $U^{\prime}$ filters) between the BHB and RGB. Moreover, it was obtained for GCs in a limited range of metallicity and with predominant BHB morphology. However, it is not clear (1) whether there are changes of the mutual positions in the $U$ and $U^{\prime}$ not only between the BHB and RGB, but also between them and the red $\mathrm{HB}$ (RHB) and turnoff point of the same GC; (2) whether there is any dependence of such changes on cluster metallicity. Therefore, we extend our study to a wider range of $U$ (and $U^{\prime}$ ) magnitudes of cluster stars and a wider range of GC metallicity, as well as to GCs exhibiting both the BHB and RHB.

\section{Observations and data reduction}

The observations were acquired on three nights, October 9/10, $10 / 11$ and $11 / 121998$, with the $1.3 \mathrm{~m}$ Warsaw telescope, Las Campanas Observatory, using a set of UBVI filters and a $2048 \times$ 2048 CCD camera with a gain $=3.8$ and a readout noise of $5.5 e^{-} \mathrm{rms}$. The array scale was $0^{\prime} .417 \mathrm{pixel}^{-1}$, giving a field of view of $14^{\prime} \times 14^{\prime}$. The center of the measured field of NGC 6864 was approximately $50^{\prime \prime}$ to the east and $10^{\prime \prime}$ to the south of the cluster center. Flat-field, bias and dark frames were taken twice per night, at the beginning and the end of each one. We took a total of 9 frames in $U$ (exposure time from $90 \mathrm{~s}$ to $360 \mathrm{~s}$ ), 9 frames in $B(60 \mathrm{~s}$ to $180 \mathrm{~s}), 9$ frames in $V(40 \mathrm{~s}$ to $120 \mathrm{~s})$, and 9 frames in $I(40 \mathrm{~s}$ to $120 \mathrm{~s})$. The average seeing estimated from the observations was about $11^{\prime \prime} 0-1$ '. 3 . Table 1 lists the $\log$ of the frames obtained.

The reductions of CCD photometry were performed at the Isaac Newton Institute, and at the European Southern Observatory, ESO, Santiago, Chile. The stellar photometry was carried out separately for all frames using DAOPHOT/ALLSTAR (Stetson 1987, 1991). The program stars were detected and measured by applying the usual procedures. To obtain the PSF, 20 to 30 stars in each frame, bright but far from saturation, were selected among those having no neighbors or defects within the
Table 1. Log of observations.

\begin{tabular}{|c|c|c|c|c|c|}
\hline Night & $\begin{array}{c}\text { Exp. } \\
\text { s }\end{array}$ & Filter & Airmass & $\begin{array}{c}\text { Seeing } \\
\text { Se }\end{array}$ & $\begin{array}{c}\text { Julian Day } \\
2451090.0+\end{array}$ \\
\hline $9 / 10$ & 40 & $\bar{V}$ & 1.201 & 1.1 & 6.58468 \\
\hline $9 / 10$ & 60 & $B$ & 1.207 & 1.1 & 6.58628 \\
\hline $9 / 10$ & 90 & $U$ & 1.215 & 1.2 & 6.58818 \\
\hline $9 / 10$ & 40 & I & 1.225 & 1.0 & 6.59000 \\
\hline $9 / 10$ & 40 & V & 1.246 & 1.1 & 6.59417 \\
\hline $9 / 10$ & 60 & $B$ & 1.253 & 1.1 & 6.59579 \\
\hline $9 / 10$ & 120 & $U$ & 1.262 & 1.2 & 6.59786 \\
\hline $9 / 10$ & 40 & $I$ & 1.276 & 1.0 & 6.59985 \\
\hline $9 / 10$ & 40 & V & 1.299 & 1.1 & 6.60394 \\
\hline $9 / 10$ & 60 & $B$ & 1.308 & 1.2 & 6.60558 \\
\hline $9 / 10$ & 120 & $U$ & 1.319 & 1.3 & 6.60767 \\
\hline $9 / 10$ & 40 & $I$ & 1.335 & 1.0 & 6.60966 \\
\hline $10 / 11$ & 40 & V & 1.247 & 1.1 & 7.59179 \\
\hline $10 / 11$ & 60 & $B$ & 1.255 & 1.1 & 7.59340 \\
\hline $10 / 11$ & 120 & $U$ & 1.264 & 1.2 & 7.59547 \\
\hline $10 / 11$ & 40 & $I$ & 1.278 & 1.1 & 7.59748 \\
\hline $10 / 11$ & 40 & V & 1.286 & 1.1 & 7.59897 \\
\hline $10 / 11$ & 60 & $B$ & 1.295 & 1.2 & 7.60058 \\
\hline $10 / 11$ & 120 & $U$ & 1.305 & 1.3 & 7.60267 \\
\hline $10 / 11$ & 40 & I & 1.320 & 1.1 & 7.60466 \\
\hline $10 / 11$ & 40 & V & 1.330 & 1.1 & 7.60615 \\
\hline $10 / 11$ & 60 & $B$ & 1.339 & 1.2 & 7.60775 \\
\hline $10 / 11$ & 120 & $U$ & 1.351 & 1.3 & 7.60984 \\
\hline $10 / 11$ & 40 & $I$ & 1.368 & 1.2 & 7.61183 \\
\hline $11 / 12$ & 120 & V & 1.234 & 1.0 & 8.58694 \\
\hline $11 / 12$ & 180 & $B$ & 1.248 & 1.1 & 8.58997 \\
\hline $11 / 12$ & 360 & $U$ & 1.265 & 1.2 & 8.59436 \\
\hline $11 / 12$ & 120 & $I$ & 1.296 & 1.0 & 8.59846 \\
\hline $11 / 12$ & 120 & V & 1.332 & 1.1 & 8.60427 \\
\hline $11 / 12$ & 180 & $B$ & 1.349 & 1.2 & 8.60725 \\
\hline $11 / 12$ & 360 & $U$ & 1.373 & 1.2 & 8.61166 \\
\hline $11 / 12$ & 120 & $I$ & 1.413 & 1.2 & 8.61573 \\
\hline $11 / 12$ & 120 & V & 1.434 & 1.2 & 8.61839 \\
\hline $11 / 12$ & 180 & $B$ & 1.456 & 1.2 & 8.62140 \\
\hline $11 / 12$ & 360 & $U$ & 1.485 & 1.3 & 8.62580 \\
\hline $11 / 12$ & 120 & I & 1.537 & 1.1 & 8.62988 \\
\hline
\end{tabular}

Table 2. Data on photoelectric standards.

\begin{tabular}{ccccccc}
\hline \hline Star & $X$ & $Y$ & $V$ & $U-V$ & $B-V$ & $V-I$ \\
\hline A & 680.8 & 799.1 & 13.650 & 0.090 & 0.720 & 0.840 \\
& & & -0.028 & -0.059 & -0.034 & -0.004 \\
B & 880.8 & 1282.6 & 13.770 & 0.170 & 0.750 & 0.840 \\
& & & 0.006 & 0.005 & -0.019 & -0.007 \\
C & 1060.6 & 1475.9 & 14.230 & 0.160 & 0.780 & 0.870 \\
& & & 0.012 & 0.035 & -0.013 & -0.023 \\
D & 803.7 & \multirow{2}{*}{1501.6} & 14.240 & 0.480 & 1.010 & 1.040 \\
& & & -0.023 & -0.040 & 0.065 & 0.070 \\
G & 1042.8 & \multirow{2}{*}{1540.3} & 15.210 & 0.280 & 0.800 & 0.820 \\
& & & 0.032 & 0.062 & 0.001 & -0.036 \\
\hline
\end{tabular}

PSF radius. We find that among standard PSFs provided by DAOPHOT, the PENNY2 function enables us to handle aberrations, specific to individual frames, most effectively. To calibrate our photometry, we relied on photoelectric standards previously set up by Alvarado et al. (1990) in the cluster field. It is the standard approach used at the Isaac Newton Institute within the framework of photometric studies of GCs. Table 2 lists the photoelectric standards used in M 75, their photoelecric magnitudes and colors with the corresponding deviations, in the sense: photoelectric values minus CCD ones. The formulae used in this 
study to bring instrumental magnitudes and colors to the standard $U B V I$ photometric system are as follows:

$V=v-0.055( \pm 0.055)(b-v)+0.020( \pm 0.041)$,

$V=v-0.025( \pm 0.038)(v-i)+0.019( \pm 0.046)$

$U-B=1.099( \pm 0.032)(u-b)-0.008( \pm 0.028)$,

$B-V=0.952( \pm 0.012)(b-v)-0.032( \pm 0.013)$,

$V-I=0.962( \pm 0.025)(v-i)+0.048( \pm 0.025)$.

In our preliminary list, we retained only those stars that had at least two measurements per night in each photometric band. We included in our final list only those stars for which at least one color index was determined. A total of 2681 stars were measured both in $V$ and $I$. Of them, 1547 and 659 stars have $B$ and $U$ magnitudes determined, respectively ${ }^{1}$. For stars of the brighter sequences, i.e. with $V<18.0$, the rms errors are, on average, 0.028 in $U, 0.021$ in $B, 0.017$ in $V$, and 0.013 in $I$.

Numerous stars measured by us in M 75 are in common with the study by Catelan et al. (2002). We found 1434 such stars. To compare both photometries, however, we used the most reliable data, namely those based on cross-identifications of the brighter stars $(V<19.0)$ in the less crowded parts of the cluster field. From a sample of 621 stars retained, we have obtained the following estimates of the mean differences between the two photometries, in the sense Catelan et al. (2002) minus the present study: $\delta V=-0.058( \pm 0.087) ; \delta(U-B)=+0.011( \pm 0.085)$; $\delta(B-V)=-0.108( \pm 0.076) ; \delta(V-I)=-0.018( \pm 0.079)$. The $(B-V)$ color difference between the photometries is larger than the corresponding differences in other colors, which are quite small. This difference becomes apparent at $(B-V)<1.3$ and $V>$ 16.0. Calibration of stellar photometry by standard stars located in observed star fields should, in principle, be more accurate than a calibration relying on external standard stars. Although we use standard stars located in the observed field of M 75, the situation is not certain. The number of the available standard stars within our field is limited (5 stars), and among them there is no star in the blue part of the color range $\Delta(B-V)$ covered by stars in the cluster CMD. For this reason, we cannot exclude systematic effects in our calibration, in particular in the color $(B-V)$.

\section{The color-magnitude diagrams}

\subsection{General comments on the cluster and field star color-magnitude diagrams}

The field of M 75 is observed to be contaminated by a number of field stars. To simultaneously obtain a larger sample of cluster stars and to reduce the effect of the field stars on the results of our photometry, we optimize the cluster-to-field star ratio in the analyzed sample of stars. We have determined the dependence of the projected density of stars in the field of M 75 as a function of the radial distance from the center of the cluster. We show this dependence in Fig. 1 for two limiting magnitudes, $I_{\mathrm{lim}}=19.5$ (red line and squares) and $V_{\mathrm{lim}}=19.0$ (green line and squares). The projected density is expressed as the number of stars per $10^{3}$ square pixels. The estimated densities converge near the

\footnotetext{
1 The results of our photometry are available at the CDS.
}

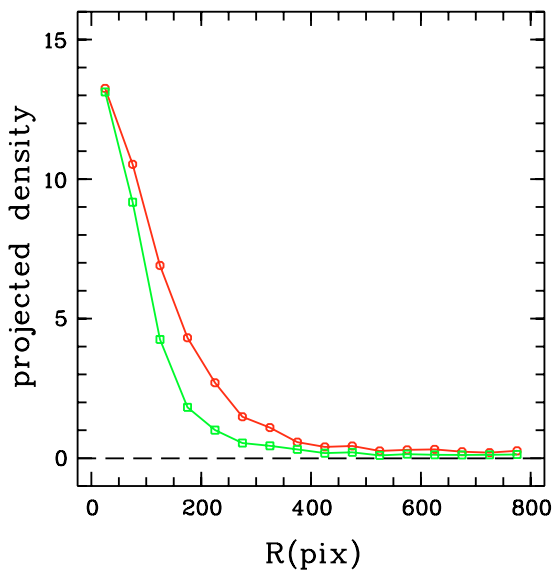

Fig. 1. The dependence of the projected density of stars in the field of M 75, expressed (on a linear scale) as the number of stars per $10^{3}$ square pixels, as a function of the radial distance from the center of the cluster; red circles and green squares connected by lines of the corresponding colors show the projected densities calculated for two limiting magnitudes, $I_{\lim }=19.5$ and in $V_{\lim }=19.0$, respectively.

cluster center because of decreasing numbers of detected faint stars in the central part due to crowding effects. However, at larger radial distance, the sample of stars with fainter limiting magnitude shows a higher projected density. The most significant changes in the projected density of stars occur within a radius $R<500$ pixels $\left(\sim 200^{\prime \prime}\right)$. With increasing distance from the cluster center, the density decreases much more gradually and asymptotically approaches its constant value. This implies that at $R>500$ pixels, the projected density of cluster stars belonging to the brighter sequences becomes comparable to that of the field stars. Hence, we accept that $R \sim 500$ pixels is the border radius to sample a major fraction of the cluster population. It should be noted, however, that this radius can vary for the cluster star belonging to different sequences or magnitude ranges, since the cluster and field stars show distinct distributions in the CMD. In particular, in the region of the cluster BHB, there are no or a very small number of field stars.

We give our photometric result for measured stars in a wide field of M 75 in three panels of Fig. 2 where the $V-(B-V)$, $I-(V-I)$ and $U-(U-B)$ CMDs are shown. Blue dots correspond to stars confined within the region with radius $R=500$ pixels, red circles show stars from the external part (with $R>1000$ pixels) of the observer field. Both cover approximately equal areas. Stars located at intermediate distances $(500<R<1000$ pixels $)$ from the cluster center are shown with small green dots.

The right panel of of Fig. 2 shows the somewhat uncommon appearance of the HB in the CMD (between $0.0<U-B<0.3$ and $18.0<U<19.0$ ) with the $U$-based color and $U$ magnitude, as compared to CMDs with widely used colors and magnitudes. The BHB and RHB fall approximately in the same color range and appear as a clump, the former being a brighter, nearly horizontal sequence of stars within this clump, and the latter as a fainter and tilted one.

The number of cluster stars in the external part should already be quite small, taking into account their radial distance from the cluster center and the expected density of the cluster stars at this distance. The real number of field stars in the CMDs is not more than the number of the stars shown by red symbols in the corresponding panels. One can see that the contamination by the field stars of the BHB and the upper RGB is negligible. A somewhat larger number of field stars can fall in the region of 

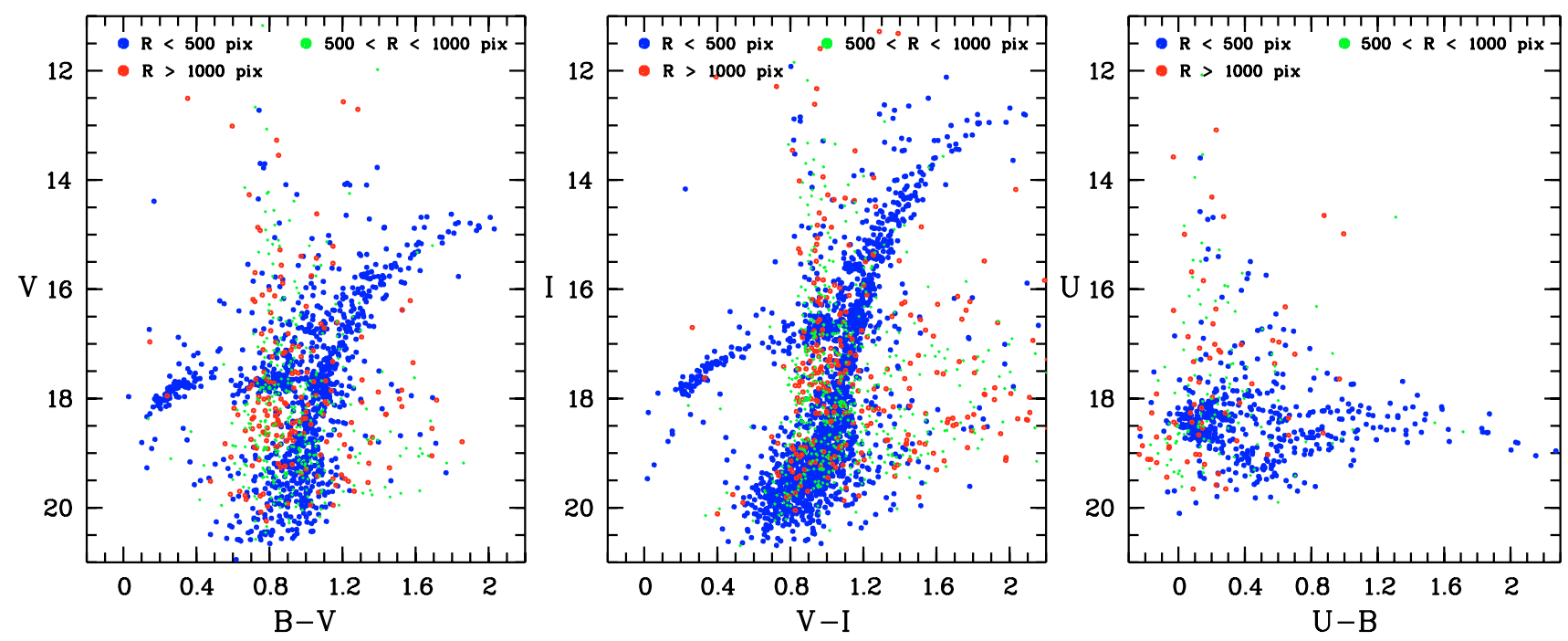

Fig. 2. The $V-(B-V), I-(V-I)$ and $U-(U-B)$ CMDs for stars in $13^{\prime} \times 13^{\prime}$ field of the Galactic GC M 75 . Blue symbols denote stars with the projected distance $R<500$ pixels $\left(\sim 200^{\prime \prime}\right)$ from the cluster center, while red ones show stars from the outer part (with $R>1000$ pixels) of the observed field, the bulk of the latter being the field stars; stars located at intermediate distance $(500<R<1000$ pixels $)$ from the cluster center are presented by small green dots.

the AGB, RHB, and RGB at the level of the HB and especially below it. The blue boundary of the field stars in our CMDs in the registered magnitude range is almost vertical and approximately corresponds to the color of the blue boundary of the RHB. The CMD of the stars from the outer part of our field is in good general agreement with the CMD of the field stars around M 75, obtained by Catelan et al. (2002). We note that the limiting $V$ magnitude achieved in our photometry is somewhat deeper than can be seen in the $V-(B-V)$ diagram because of a shallower limit in the $B$ bandpass. In the $V-(V-I)$ diagram, that is not shown in Fig. 2, the limiting magnitude is very close to the magnitude of the turnoff point occurring, according to Catelan et al. (2002), at $V=21.22$.

While the demonstrated CMDs include all the measured stars with $R<500$ pixels, in our subsequent analysis of the photometry we have excluded stars located in the region with $R<40$ pixels $\left(\sim 16^{\prime \prime}\right)$ where photometric accuracy decreases appreciably.

\subsection{The asymptotic and red giant branches}

Our photometry of M 75 yielded a fairly large sample of stars belonging to the evolved sequences. This allowed us to address the main features of the star distribution on the AGB and RGB.

The CMDs in Fig. 2 show that the number of stars tracing the AGB, at least in its most populated lower part, in the magnitude range $\Delta V \approx 1.5$ mag makes it possible to unambiguously identify an important feature, the so-called AGB clump at the base of the branch. It is seen at $V \approx 16.8$ and in the color range $0.95 \leq(B-V) \leq 1.10$ in the $V-(B-V)$ diagram. In the $I$ - $(V-I)$ diagram, the clump is seen at $I \approx 15.6$. It is more evident in the latter diagram thanks to its narrower color range, $1.10 \leq(V-I) \leq 1.20$.

The formation of the AGB clump, as well as the well-known RGB bump, is caused by a slowing down of the rate of stellar evolution along the given evolutionary sequence(s). For more details concerning the nature of the clump and useful parameters deduced for it from the CMD, as well as for more references related to the subject, we refer, in particular, to Ferraro et al. (1999) and Sandquist \& Bolte (2004). In the present paper, we have been able to estimate one of the parameters, namely the difference between the $V$-levels of the HB and the AGB clump, $\Delta V_{\mathrm{HB}}^{\text {clump }}$. Ferraro et al. (1999) note that the estimations of the given parameter are available for very limited number of GCs, since the AGB is poorly populated. Also, to avoid any ambiguity in deducing this magnitude difference, they argue to rely on the level of the zero-age HB (ZAHB) and point out that "one might ideally define the ZAHB level by adopting the magnitude of the lower envelope of the observed HB distribution in the region with $0.2<B-V<0.6^{\prime \prime}$, i.e. red-ward of the blue boundary of the instability strip. We followed the same procedure and found $V_{\mathrm{HB}}=17.82 \pm 0.03$. In turn, $V_{\text {clump }}=16.75 \pm 0.03$ that leads to $\Delta V_{\mathrm{HB}}^{\text {clump }}=1.07 \pm 0.06$.

To obtain the RGB luminosity function (RGBLF) of M 75, we avoid contamination of the RGB by (1) stars belonging to both the AGB and RHB, (2) stars showing large deviation from the sequence's fiducial line due to photometric error or (3) possible field stars that appear among the RGB stars on one CMD, but are displaced from the RGB on another CMD. We used the advantage of multi-color photometry and proceeded in the following way. In each of the CMDs, $V-(B-V)$ and $I-(V-I)$, we fitted the mean locus of the RGB with a polynomial using the corresponding commands in the MIDAS system. We next linearized the RGB by subtracting for each star the color of the mean locus at its luminosity level from the star's color index. To construct the RGBLF, we used only those stars that satisfied our selection criterion: their deviations, $\delta(B-V)$ and $\delta(V-I)$, from the mean locus in both colors simultaneously did not exceed $\pm 0.06 \mathrm{mag}$. On the one hand, this value is close to mean error in the colors of the fainter RGB stars, i.e. at the level of the HB and below it, and somewhat larger than the mean error of the brighter RGB stars. On the other hand, this conditional boundary of the RGB separates the bulk of its stars from the majority of stars belonging to the asymptotic and red horizontal branches.

The obtained RGBLF of M 75 is shown by the blue line in the upper panel of Fig. 3. For a more reliable analysis, we compare this LF with the analogous one (green line, lower panel) obtained by Alcaíno et al. (1998b) in the GC M 80. Moreover, the generalized RGBLF (red line) from Kravtsov (1989) is shown 


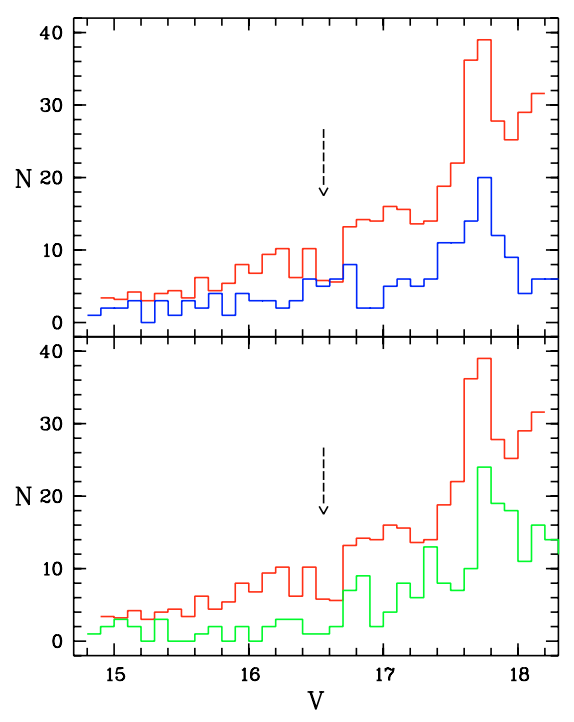

Fig. 3. The RGBLF of M 75 (blue line, upper panel) is compared with its counterpart obtained for M 80 (green line, lower panel) by Alcaino et al. (1998b), as well as with the generalized RGBLF (red line) taken from Kravtsov (1989). The two latter LFs are shown in the magnitude range of the former one, and they are shifted along the $V$-axis until the coincidence of the bump position of all the LFs. The generalized RGBLF is scaled down by a factor of 5 as compared to the original one. Arrow indicates the gap present in the RGBLFs of many GCs.

in both panels. It has been scaled by a factor of 5 as compared to the original one. Also, this LF and RGBLF of M 80 have been reduced to the magnitude range of the RGBLF of $M 75$ by shifting them along the $V$-axis until reaching coincidence of the bump position of all the LFs. The bump of the RGBLF of M 75 is seen at $V_{\text {bump }}=17.75 \pm 0.05$, thanks to the sizable sample of RGB stars isolated in the region of the bump. Taking into account the estimated level of the ZAHB at $V_{\mathrm{HB}}=17.82 \pm 0.03$, we obtain a value for the magnitude difference between the ZAHB and the bump $\Delta V_{\mathrm{HB}}^{\text {bump }}=0.07 \pm 0.08$. It is in good agreement with $\Delta V_{\mathrm{HB}}^{\text {bump }}=0.05$ obtained by Catelan et al. (2002). This implies virtually the same (within the uncertainty) cluster metallicity estimated using the parameter $\Delta V_{\mathrm{HB}}^{\text {bump }}$. Formally, our estimate is a few hundredth dex more metal-poor than the one deduced by Catelan et al. (2002), i.e., $[\mathrm{Fe} / \mathrm{H}]_{Z W} \approx-1.33 \mathrm{dex}$ in the scale of Zinn \& West (1984).

The RGB bump is the only RGBLF feature that is widely accepted to be physically real and in any GCs. However, in the middle of 1980s one sometimes failed to detect this feature, particularly in a number of the most metal-poor GCs in which it was systematically less pronounced than in more metal-rich GCs. As for other physically real features on RGBLFs of GCs, their existence is still uncertain. Kravtsov (1989) not only showed, for the first time, the dependence of the RGB bump position in $V$ magnitude on GC metallicity, but also studied the problem of the existence of other possible RGB features. He used a generalized RGBLF obtained from a sample of the LFs of the upper RGB parts of GCs belonging to the metal-poor range. He concluded that the bump is probably not the only real feature common to the RGB of different GCs. The generalized RGBLF has revealed three additional, statistically significant features that can be related to the peculiarities of the evolution of stars along the RGB of the clusters. At least one of them is especially pronounced. It shows up as a quite significant gap on the generalized RGBLF, and systematically appears in individual LFs as a depression or abrupt change in the LFs. Its statistical significance in the generalized RGBLF was estimated and discussed in Kravtsov (1989). Specifically, the probability (P) that this feature in the generalized RGBLF is a result of statistical fluctuation is $P<0.001$. The position in $V$ magnitude of the gap under consideration is, on average, around $1.1 \pm 0.1$ above the bump. It is marked by rows in both panels of Fig. 3. This feature was also noted by us in the RGBLF of the Small Magellanic Cloud populous star cluster Kron 3 at $V \sim 18.5$ (Alcaíno et al. 1996a) and in the RGBLF of M 80 at $V \sim 14.2$ (Alcaíno et al. 1998b). In the magnitude range of the RGBLF of M 75 it corresponds to $V \sim$ 16.6. The RGBLF of M 75 itself does not show any significant gap at the given position. More exactly, the RGBLF sampled with a smaller bin, $\delta V=0.05$, does show a deep but narrow gap at this position. However, the general behavior of the RGBLF of M 75 in the magnitude range $\Delta V \approx 2.0$ mag above the bump is peculiar as compared to that of the generalized LF, in the sense that they appear to be in opposite "phase" to each other. Indeed, the former LF exhibits two depressions where the latter one shows "normal" level and a small local bump in the corresponding magnitude ranges (at $16.8<V<17.4$ and $16.0<V<$ 16.3 , respectively), and vice versa, i.e. at the location of the discussed gap we see in the RGB of different GCs, the RGB of M 75 rather has a local excess of stars. Such a behavior of the RGBLF of M 75 could explain a deficit (if any) of the brighter RGB stars in M 75, noted earlier by Harris (1975) and discussed and interpreted by Catelan et al. (2002).

\subsection{The horizontal branch position in the CMDs with $U$ and $U^{\prime}$ magnitude axes}

As a first step in examining the position of the HB in the CMDs with the UV magnitude axis, we compare the corresponding CMD of M 75 with the analogous deep diagrams of NGC 288 and NGC 6723, GCs whose metallicities are confined in a limited range. The majoroty of GCs, for which we have previously obtained deep photometry reaching the TO in the UV range, were observed with the EMMI camera of the NTT/ESO telescope in 1993. NGC 288 is among these clusters (Alcaíno et al. 1997c). In turn, photometry of NGC 6723 (Alcaíno et al. 1999), with limiting $U$ magnitude below the TO as well, is based on observations gathered with the $100^{\prime \prime}$ telescope of the Las Campanas Observatory. As was established earlier and noted above, in Sect. 1, photometry (above the TO in UV) with the same telescope and equipment in both NGC 6541 (Alcaíno et al. 1997b) and M 80 (Alcaíno et al. 1998b), on the one hand, and photometry in the GC M 79 (Kravtsov et al. 1997) observed with the NTT, on the other hand, revealed disagreement in the position of the BHB in the clusters' CMDs with UV magnitude axis. For this reason, we use such diagrams for NGC 288 and NGC 6723 , as templates of deep $U-(B-V)$ and $U^{\prime}-(B-V)$ CMDs of GCs with close metallicity and different HB morphology in order to compare them to each other, as well as both to the corresponding CMD of M 75.

We superimposed the $V-(B-V)$ and $U\left(U^{\prime}\right)-(B-V)$ CMDs of NGC 288 and NGC 6723. The offsets along the luminosity axes were determined by best coincidence of the diagrams around the turnoff points. The optimal offsets along the color axes were achieved by the best matching the positions of the turnoff points and the lower RGBs. We then superimposed these matched CMDs and the same CMDs of M 75. Since the diagrams of this GC do not reach the TO, we relied on the optimal coincidence, in the magnitudes and color, of the brighter sequences of the CMDs of M 75 and NGC 6723. Specifically, the 

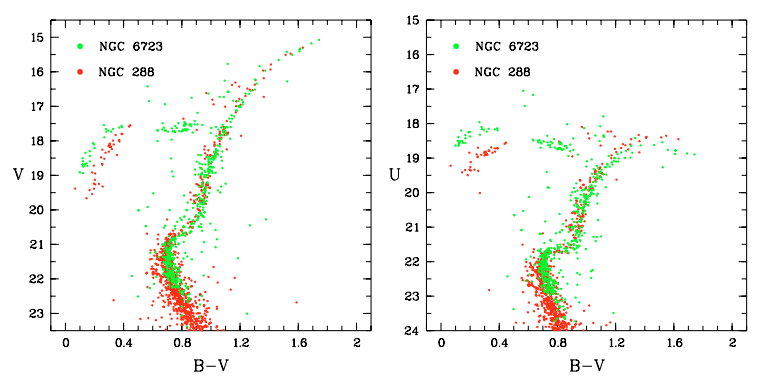

Fig. 4. The superposition of deep $V-(B-V)$ and $U\left(U^{\prime}\right)-(B-V)$ diagrams of NGC 288 and NGC 6723, achieved by matching the CMDs in the region of the turnoff point; both the magnitudes and colors of the original CMDs are reduced to those of the corresponding CMDs of NGC 6864 (M 75).
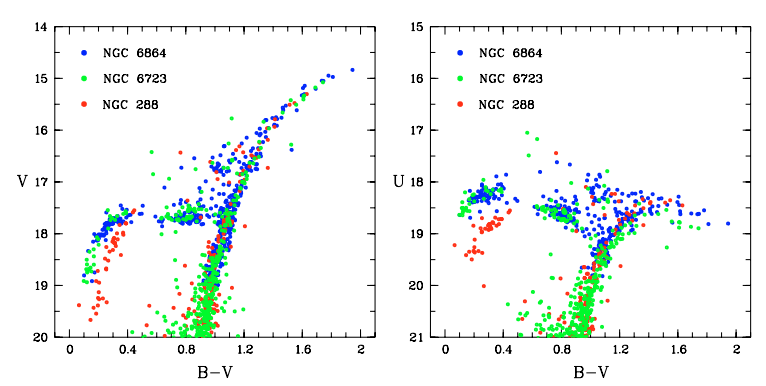

Fig. 5. The superposition of upper parts of the $V-(B-V)$ and $U\left(U^{\prime}\right)-(B-V)$ diagrams of NGC 288, NGC 6723 and NGC 6864 (M 75).

mean levels of the BHB and RHB were optimized in $V$ and $U$. The mean position in the $(B-V)$ color of these branches and also of the RGB at the level of the HB were used to define the offset in the color. As for the offset along the $U$-axis between the CMDs of M 75 and NGC 6723, the validity of this procedure is justified by the realization, at the $100^{\prime \prime}$ and $1.3 \mathrm{~m}$ Warsaw telescopes, of $U$ bandpass close to the standard one, i.e. encompassing the Balmer jump. In particular, according to data kindly provided by A. Udalski on a response curve of the $U$ bandpass realized at the $1.3 \mathrm{~m}$ Warsaw telescope, the wavelength of its maximum coincides with that of the standard curve. Blue-ward of their maxima, the former curve shows a somewhat lower level in comparison with the latter curve, and both curves show nearly the same transmission in the region of the Bulmer jump.

The resulting superpositions of (1) the CMDs of NGC 288 and NGC 6723 in a wide luminosity range, from the main sequence to the RGB tip, and of (2) the two GCs and M 75 in the region of the brighter sequences, are presented separately in Figs. 4 and 5. In both figures, the magnitudes and colors of the original CMDs of NGC 288 and NGC 6723 are reduced to those of the corresponding CMDs of M 75. Their TO points are at $V_{\mathrm{TO}}=21.20 \pm 0.05$, in good agreement with $V_{\mathrm{TO}}=21.22 \pm$ 0.09 defined by Catelan et al. (2002) for M 75. As seen from the $V-(B-V)$ plots, the RGBs of the three GCs are virtually indistinguishable at the level of scatter and the uncertainty of the superposition of the diagrams, and the slope of the upper parts of the cluster RGBs is identical. Also, $V$-levels of the cluster BHBs near the blue edge of the instability strip are the same within the uncertainty. It cannot be excluded that a better coincidence of the BHBs and RHBs of NGC 6723 and M 75 in the $V-(B-V)$ diagram would be for slightly lower, by $\Delta V=0.05 \mathrm{mag}$, positions of the CMD of NGC 6723 (in this case its upper RGB would be slightly lower as well). However, the $U-(B-V)$ plots show
Table 3. Color and magnitude offsets applied to the compared CMDs.

\begin{tabular}{lcccl}
\hline \hline Cluster & $\Delta U$ & $\Delta V$ & $\Delta(B-V)^{a}$ & cluster photometry \\
\hline NGC 288 & 2.70 & 2.30 & 0.220 & Alcaíno et al. (1997c) \\
NGC 1841 & -1.25 & & 0.225 & Alcaíno et al. (1996b) \\
NGC 1904 & 2.00 & & 0.300 & Kravtsov et al. (1997) \\
NGC 6397 & 5.15 & & 0.150 & Alcaíno et al. (1997a) \\
NGC 6723 & 2.50 & \multirow{2}{*}{2.15} & 0.130 & Alcaíno et al. (1999) \\
NGC 7099 & 3.15 & & 0.240 & Alcaíno et al. (1998a) \\
\hline
\end{tabular}

${ }^{a}$ The CMDs are offset to the same reference points in the system of the corresponding CMDs of $\mathrm{M} 75$; the offsets are in the sense: $(B-V)_{\mathrm{M} 75}-(B-V)_{\mathrm{GC}}$ and $\operatorname{mag}_{\mathrm{M}} 75-\operatorname{mag}_{\mathrm{GC}}$

obvious differences between $U^{\prime}$ - and $U$-levels of the BHB near the edge, i.e. between the BHB level of NGC 288, on the one hand, and that of M 75 and NGC 6723, on the other hand. In agreement with our previous findings, this difference is of the order of $\Delta U \sim 0.4$ to $0.5 \mathrm{mag}$. At the same time, while the RGBs of NGC 288 and M 75 almost entirely coincide with each other and show only small differences at their highest $U$-level (at their inflection point), the RGB of NGC 6723 begins to systematically deviate from the RGBs of these two clusters at $U<19.5$. The disagreement in $U$ magnitude between the RGBs relative to the $U$ magnitudes of the corresponding TO points becomes notable around the inflection points. In comparison with the variation of the $U$-level of the $\mathrm{BHB}$, this difference is mainly due to cluster metallicity.

To study the dependence of the $U\left(U^{\prime}\right)$-level of the RGB on metallicity and to trace the BHB and define its position more reliably in the $U^{\prime}-(B-V)$ diagram (because of some uncertainty in the position of the BHB of NGC 288 near the blue edge of the instability strip) we used our photometry for a sample of more metal-poor GCs. The magnitudes and colors of their CMDs have been also reduced to those of the corresponding CMD of M 75 . The data on the offsets applied to the original CMDs of all the GCs, as well as the references to the sources of the original photometric data are listed in Table 3. The superimposed CMDs are presented in Fig. 6. Here, the location of BHB in $U^{\prime}$ is reliably and unambiguously shown by additional BHB stars of a number of GCs with photometry reaching the TO points in $U^{\prime}$, namely of NGC 1904, NGC 6793, NGC 7099.

Finally, a result obtained several decades ago should be. In order to overcome a number of disadvantages of the standard $U$ bandpass that are related to the presence in it of the Balmer jump, Straižys (1973) revised this bandpass and used a UV-filter with a transmission curve blue-shifted of the jump. The resulting revised UV bandpass, designated as "W", did not include the Bulmer jump. In this sense, it is belongs to the bandpasses conditionally denoted as $U^{\prime}$. The author showed that the relation between the color indexes $(U-B)_{0}$ and $(W-B)_{0}$ was essentially nonlinear in a certain range of the color $(B-V)_{0}$, with the maximum difference between the former two indexes reaching around $(B-V)_{0}=0$, i. e., just in the region of the BHB. These and many other data on various photometric systems were summarized by Straižys (1977).

\subsection{A sensitive indicator of metallicity in the CMDs with the UV magnitude axis}

Along with the bimodal appearance of the BHB in the CMD with the UV magnitude axis, Fig. 6 shows the position of the $U\left(U^{\prime}\right)$-level of the upper RGB in GCs of different metallicity. This level is defined unambiguously. It corresponds to the 


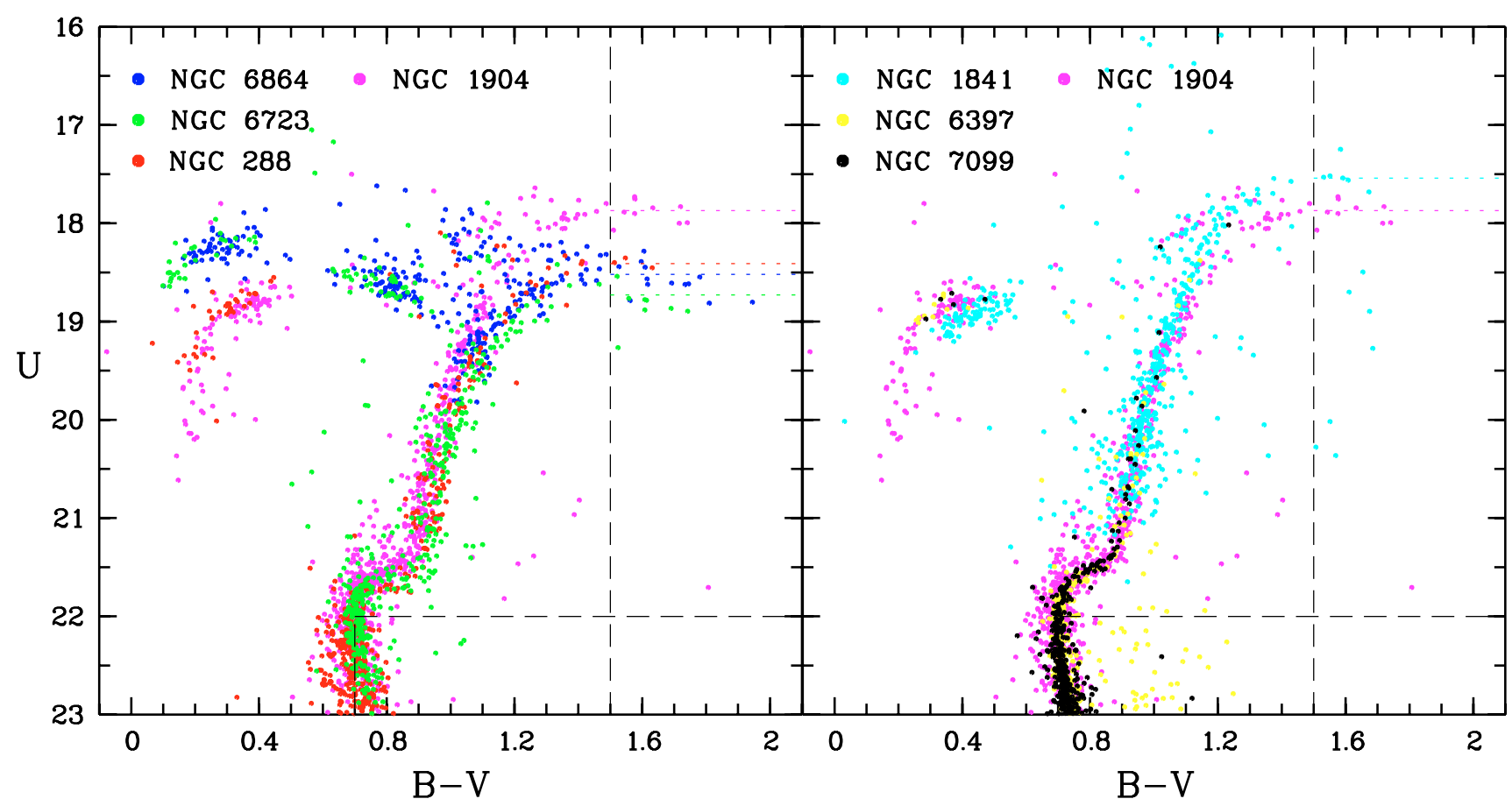

Fig. 6. Left panel: the superposition of the $U\left(U^{\prime}\right)-(B-V)$ diagrams of NGC 1904 (M 79) and of more metal-rich GCs: NGC 288, NGC 6723 and NGC 6864 (M 75); right panel: the superposition of the $U^{\prime}-(B-V)$ diagrams of NGC 1904 (M 79) and of more metal-poor GCs: NGC 1841, NGC 6397 and NGC 7099 (M 30) the $U\left(U^{\prime}\right)$-levels of inflection (brightest) point of the cluster RGBs are shown by dashed lines of the same color as the corresponding symbols of the cluster CMDs. Both the magnitudes and colors of the original CMDs are reduced to those of the corresponding CMD of M 75

inflection point of the RGB, the brightest point of the branch in UV. Only AGB stars can produce some confusion in the case of small number of brighter RGB stars. We defined the parameter $\Delta U_{\mathrm{TO}}^{\mathrm{RGB}}$ as the difference in $U\left(U^{\prime}\right)$ magnitude between the TO point and the inflection point of the RGB. The color difference $\Delta(B-V)$ between these points is around $\Delta(B-V) \approx 0.8$ and almost independent of metallicity. The mean location in the color of the inflection points of the RGBs is shown by the vertical long-dashed line. Color dashed lines show the RGB levels defined for the corresponding GC. The horizontal long-dashed line is drawn at the level of the TO point of the superimposed CMDs.

The choice of the parameter $\Delta U_{\mathrm{TO}}^{\mathrm{RGB}}$ is clear. We only note that in contrast to the UV-level of the HB (e.g., relative to that of the RGB), the UV-level of the TO point does not apparently depend on the effect under consideration. This is mainly due to the strong dependence of the Balmer jump on the temperature. It decreases rapidly with decreasing temperature and disappears (nearly) completely in stars of metal-poor GCs with temperature close to that of the main-sequence TO point stars and in cooler RGB stars. For this reason, $\Delta U_{\mathrm{TO}}^{\mathrm{RGB}}$ should not show a dependence, to a certain extent, on the position of UV-bandpass relative the Balmer jump.

In our data base, among the most metal-poor GCs with available photometry in UV, the Large Magellanic Cloud GC NGC 1841 is the only GC with a large number of brighter RGB stars measured in this bandpass. At the same time, the limiting magnitude of this photometry does not reach the TO point of the cluster. For this reason, as in the case of M 75, we offset the CMD of NGC 1841 along the $U^{\prime}$-axis until coincidence of the mean $U^{\prime}$-level of its BHB and that of the BHBs of metal-poor GCs for which deep photometry reaching the TO point in $U^{\prime}$ is available (right panel of Fig. 6). NGC 1841 is assumed to be as old as the Galactic old metal-poor GCs. The offset along the color axis was determined by the best coincidence of the lower RGB of NGC 1841 and other most metal-poor GCs.

In Fig. 7, we demonstrate, for the first time, the dependence of the parameter $\Delta U_{\mathrm{TO}}^{\mathrm{RGB}}$ on cluster metallicity, $[\mathrm{Fe} / \mathrm{H}]_{Z W}$, obtained for the set of metal-poor GCs. Cluster metallicities (and the corresponding errors) are taken from Zinn \& West (1984) for all the clusters, except NGC 1841. Its metallicity value is from Ferraro et al. (1999). The long-dashed line is a polynomial fit to the data, assuming a linear relation between them. From this fit, we derive the following simple relation between $\Delta U_{\mathrm{TO}}^{\mathrm{RGB}}$ and $[\mathrm{Fe} / \mathrm{H}]_{Z W}$ in the metal-poor range:

$[\mathrm{Fe} / \mathrm{H}]_{Z W}=1.5-0.8 \Delta U_{\mathrm{TO}}^{\mathrm{RGB}}$.

Figure 7 shows a very tight correlation between these values and the surprisingly small dispersion of the data. Note that it becomes somewhat larger for the case of metallicity values derived (for some clusters) from high-resolution spectroscopy or taken from the Harris catalog (Harris 1996). The differences in metallicity between M 75, NGC 288, NGC 6723, estimated from the above relation, using values of the parameter $\Delta U_{\mathrm{TO}}^{\mathrm{RGB}}$, formally nearly coincide with those determined by relying on the data from Zinn \& West (1984).

The parameter $\Delta U_{\mathrm{TO}}^{\mathrm{RGB}}$ is approximately twice as sensitive to metallicity variation than the position of the bump on the RGB relative to the HB level in $V$ magnitude.

Table 4 lists the GCs of our set, their metallicities, estimated values of the parameter $\Delta U_{\mathrm{TO}}^{\mathrm{RGB}}$, as well as errors of these characteristics.

Strictly speaking, the parameter $\Delta U_{\mathrm{TO}}^{\mathrm{RGB}}$ for a given GC depends on the reddening of the cluster. Indeed, $\Delta U_{\mathrm{TO}}^{\mathrm{RGB}}$ relies on $U$ magnitudes of stars belonging to different luminosity classes and spectral types. In turn, extinction of stars in any bandpass is 


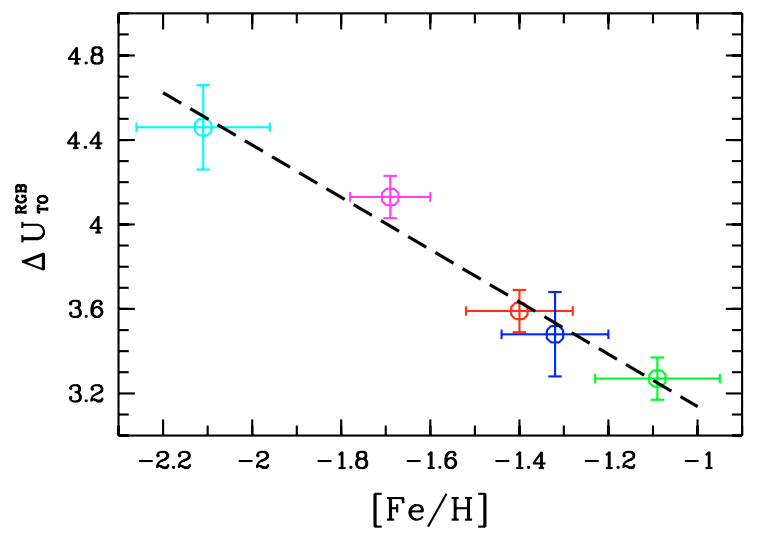

Fig. 7. The dependence of the parameter $\Delta U_{\mathrm{TO}}^{\mathrm{RGB}}$ on cluster metallicity, $[\mathrm{Fe} / \mathrm{H}]_{Z W}$; long-dashed line is a polynomial fit to the data, accepting a linear relation between them. Colors of symbols and of error bars are the same GCs as in the previous figure.

Table 4. Data on the parameter $\Delta U_{\mathrm{TO}}^{\mathrm{RGB}}$ and metallicities $[\mathrm{Fe} / \mathrm{H}]_{Z W}$ of GCs.

\begin{tabular}{lcccc}
\hline \hline Cluster & $\Delta U_{\mathrm{TO}}^{\mathrm{RGB} a}$ & $\sigma_{\Delta U}$ & {$[\mathrm{Fe} / \mathrm{H}]_{Z W}$} & $\sigma_{[\mathrm{Fe} / \mathrm{H}]}$ \\
\hline NGC 288 & 3.59 & 0.10 & -1.40 & 0.12 \\
NGC 1841 & $(4.46)$ & 0.20 & -2.11 & 0.15 \\
NGC 1904 & 4.13 & 0.10 & -1.69 & 0.09 \\
NGC 6723 & 3.27 & 0.10 & -1.09 & 0.14 \\
NGC 6864 & $(3.48)$ & 0.20 & -1.32 & 0.12 \\
\hline
\end{tabular}

$a$ The values indicated in parentheses mean that cluster photometry does not reach the main-sequence turnoff point.

a function of their temperature (spectral type), luminosity class, and abundance (e.g., Grebel \& Roberts 1995, and references therein). Therefore, the $\Delta U_{\mathrm{TO}}^{\mathrm{RGB}}$ deduced is affected by the different dependence of extinction $A_{U}$ of the RGB and main sequence TO stars on their reddening $E(B-V)$. For this reason, we estimate the effect of reddening on the values of $\Delta U_{\mathrm{TO}}^{\mathrm{RGB}}$ defined for the GCs in our study. With this aim we used the calculations obtained in Grebel \& Roberts (1995) and presented, in particular, in their Tables 1 and 4, as well as in Figs. 4 and 5. These calculations "produce ratios of the passband and colour extinctions to $A_{V, 0}$, the $V$ band extinction of a MS star with a temperature of $17000 \mathrm{~K}$ and solar metallicity". $A_{V, 0}$ is defined to be 1.0. According to the Harris catalog (Harris 1996), NGC 1904 and NGC 6864 have the smallest $[E(B-V)=0.01]$ and largest $[E(B-V)=0.16]$ reddening, respectively, among the GCs of our sample. This means that the differences of the reddening and $V$ band extinction between the two GCs are $\Delta E(B-V)=0.15$ and $\Delta A_{V} \approx 0.5$, respectively. The difference in metallicity between NGC 6864 and NGC 1904 is around $\Delta[\mathrm{Fe} / \mathrm{H}] \approx 0.5$ dex. The effective temperatures and surface gravities for the stars at the main sequence TO and RGB inflection points are accepted to be $T_{\text {eff }} \approx 6750 \mathrm{~K}, \log g \sim 5.0$ and $T_{\text {eff }} \approx 4250 \mathrm{~K}, \log g \sim 2.5$. For stars with such characteristics in the two clusters, we estimate $\delta\left[\left(\Delta U_{\mathrm{TO}}^{\mathrm{RGB}}\right)_{N 1904}-\left(\Delta U_{\mathrm{TO}}^{\mathrm{RGB}}\right)_{N 6864}\right]=\left(\Delta A_{U, \mathrm{TO}}-\Delta A_{U, \mathrm{RGB}}\right) \sim 0.01$ or so, where $\triangle A_{U \text {,TO }}$ is the difference in the $U$ band extinction of main sequence TO stars in NGC 6864 and in NGC 1904, and $\Delta A_{U, \mathrm{RGB}}$ is the same for stars at the RGB inflection points of the same GCs. The quantity $\delta$ shows how many of the differences between $\Delta U_{\mathrm{TO}}^{\mathrm{RGB}}$ of the two clusters would change if reddening of NGC 6864 was reduced to that of NGC 1904. It is at least an order of magnitude lower than the typical error of the parameter under consideration. Therefore, it cannot affect the dependence deduced here between $\Delta U_{\mathrm{TO}}^{\mathrm{RGB}}$ and $[\mathrm{Fe} / \mathrm{H}]$. For the $U^{\prime}$ bandpass, the above-estimated effect is of the same order of magnitude, i.e. it is small compared to the the typical error of $\Delta U_{\mathrm{TO}}^{\mathrm{RGB}}$.

The impact of differences in the response curves of differently realized $U$ bandpass on the dependence of the corresponding extinction $A_{U}$ on reddening $E(B-V)$ is another factor that can affect relative positions of stars in $U$ magnitude, in particular $\Delta U_{\mathrm{TO}}^{\mathrm{RGB}}$ or $\Delta U_{\mathrm{TO}}^{\mathrm{BHB}}$, the $U$ magnitude difference between the main sequence $\mathrm{TO}$ and $\mathrm{BHB}$. The larger variations of the difference between extinction $A_{U^{\prime}}$ and $A_{U}$ as a function of $E(B-V)$ among stars belonging to distinct spectral types and luminosity classes, the larger the variations between $\Delta U$ and $\Delta U^{\prime}$, defined for these stars, as a function of reddening. In other words, if the difference between $A_{U^{\prime}}[E(B-V)]$ and $A_{U}[E(B-V)]$ was the same for stars of any spectral type and luminosity class, the impact of the factor under consideration would be zero. As an example, we compare the difference between $A_{U^{\prime}}[E(B-V)]$ and $A_{U}[E(B-V)]$ for the ultraviolet bandpass realized with the $F 336 W$ filter at HST and the standard $U$ bandpass, respectively. The transmission curve of F336W (e.g., Holtzman et al. 1995) is notably blue-shifted of the Bulmer jump. So, the corresponding ultraviolet bandpass is an example of the conditionally defined $U^{\prime}$ bandpass. Holtzman et al. (1995) have presented in their Fig. 12 "a plot of extinction for several WFPC2 filters and for $U B V R I$ as function of $E(B-V)$ up to $E(B-V)=1.0^{\prime \prime}$, using synthetic computations with $\mathrm{O} 6$ and $\mathrm{K} 5$ input spectra. In turn, Tables $12 \mathrm{a}$ and $\mathrm{b}$ from the paper contain the same data, but for WFPC2 filters alone. The difference between extinction $A_{F 336 W}$ and $A_{U}$ is insignificant at low reddening for the early spectral type O6. It is of the order of a few hundredth of a mag at $E(B-V)=0.15$. For late spectral types K5, however, this difference is larger with opposite sign. Apart from this very approximate evaluation we obtained more rigorous estimates. We calculated $A_{U}$ as a function of $E(B-V)$, using analytical expressions deduced by Ažusienis $\&$ Straižys (1966), and data from their Tables 1 and 2 . We found that $A_{F 336 W}(\mathrm{O} 6)-A_{U}(\mathrm{O} 7) \approx 0.03$ at $E(B-V)=0.15$ for the early stars. For the late spectral type K5 and at the same reddening, $A_{F 336 W}(\mathrm{~K} 5)-A_{U}(\mathrm{~K} 5) \approx-0.18$. These results are in reasonable agreement with what can be estimated from the graphically presented dependence of the extinction $A_{U}$ and $A_{F 336 \mathrm{~W}}$ on $E(B-V)$ by Holtzman et al. (1995). This change of the dependence of the extinction $A_{F 336 W}$ on $E(B-V)$ for the K5 spectral type is mainly due to the fairly significant red leak of $F 336 \mathrm{~W}$. The red leak slightly affects the dependence of the $U\left(U^{\prime}\right)$ band extinction on $E(B-V)$ for early stars, but its impact can be significant in the case of late stars, especially with large amounts of absorbing matter (e.g., Ažusienis \& Straižys 1966). Therefore, when reddening increases, the parameter $\Delta U_{\mathrm{TO}}^{\mathrm{RGB}}$ determined from observations with an ultraviolet filter having (significant) red leak is expected to have increasing systematic error (comparable to the random error or exceeding it), as compared to the same parameter obtained using $U$ or $U^{\prime}$ filters without red leak. The same effect (but somewhat less strong and with opposite sign) should affect the magnitude difference $\Delta U_{\mathrm{TO}}^{\mathrm{BHB}}$, as well.

\section{Conclusions}

We obtained new multi-color photometry of more than 2600 stars in a wide field of the southern GC M 75, above its turnoff point. We used this photometry to analyze the brighter sequences of CMD.

We were able to isolate, for the first time, the AGB clump and to determine the parameter $\Delta V_{\mathrm{HB}}^{\text {clump }}=1.07 \pm 0.06$, the difference in $V$ magnitude between the ZAHB level and that of the 
clump. Also, by relying on the obtained LF of the upper RGB, we estimated similar parameter for the RGB bump, $\Delta V_{\mathrm{HB}}^{\mathrm{bump}}=$ $0.07 \pm 0.08$, as well as the behavior and features of the LF.

We combine the present $U$ photometry of M 75 with our analogous photometric data on metal-poor GCs, $[\mathrm{Fe} / \mathrm{H}]_{Z W}<$ -1.1 dex, obtained from observations with different equipment, but calibrated by standard stars located in the observed cluster fields. We investigate the position of the HB in deep CMDs reaching the turnoff point, with UV magnitude axis. We demonstrate that the difference in $U$ magnitude, $\Delta U_{\mathrm{TO}}^{\mathrm{BHB}}$, between the TO point and the $U$-level of the blue HB near the blue edge of the instability strip is bimodal. The BHB of GCs observed with the EMMI camera of the NTT/ESO telescope is about 0.4-0.5 mag smaller as compared to GCs observed with the $100^{\prime \prime}$ telescope and $1.3 \mathrm{~m}$ Warsaw telescope of the Las Campanas Observatory. However, another parameter, $\Delta U_{\mathrm{TO}}^{\mathrm{RG}}$, the difference in $U$ magnitude between the TO point and inflection point of the RGB, does not obviously depend on the characteristics of $U$ filters used, at least within insignificant reddening and provided that the filters do not have significant red leak. It shows very tight, nearly perfect correlation with cluster metallicities, $[\mathrm{Fe} / \mathrm{H}]_{Z W}$, taken from Zinn \& West (1984). We deduce, for the first time, an analytical relation between $\Delta U_{\mathrm{TO}}^{\mathrm{RGB}}$ and $[\mathrm{Fe} / \mathrm{H}]_{Z W}$.

From the point of view of practical application of the demonstrated sensitive indicator of metallicity, especially in the case of photometry of resolved stars in GCs populating near galaxies, one must apply a slightly modified approach. Specifically, the parameter $\Delta U_{\mathrm{BHB}}^{\mathrm{RGB}}$ (the difference in $U$ magnitude between the BHB level and inflection point of the RGB) is more easily measurable than $\Delta U_{\mathrm{TO}}^{\mathrm{RGB}}$. Moreover, the former is presumably less dependent on cluster age than the latter. Formally, it is quite easy to transform the obtained relation between $\Delta U_{\mathrm{TO}}^{\mathrm{RGB}}$ and $[\mathrm{Fe} / \mathrm{H}]_{Z W}$ into the relation between $\Delta U_{\mathrm{BHB}}^{\mathrm{RGB}}$ and $[\mathrm{Fe} / \mathrm{H}]_{Z W}$ by accepting the same slope for both relations. For this purpose, it is sufficient to take into account the difference in $U\left(U^{\prime}\right)$ magnitude between the TO point and $U$-level of the BHB: $\Delta U_{\mathrm{BHB}}^{\mathrm{RGB}}=\Delta U_{\mathrm{TO}}^{\mathrm{RGB}}-3.8$ and $\Delta U_{\mathrm{BHB}}^{\prime \mathrm{RGB}}=\Delta U_{\mathrm{TO}}^{\mathrm{RGB}}-3.3$ for the $U$ and $U^{\prime}$ bandpasses, respectively. Here, the $U$-level of the BHB is accepted in the same sense as proposed by Ferraro et al. (1999) and used by us above to define the $V$-level of the HB. However, these estimates are very preliminary. To achieve more reliable conclusions, more observational data on other GCs in the metal-poor range are required. In analogy with $V$-level of the $\mathrm{BHB}$, one can expect that it is difficult to reliably define the $U$-level of the BHB for some GCs, particularly for those clusters, like M 13, that exhibit both pure BHB morphology and an extended BHB tail. Note also that due to its larger color base the parameter $\Delta U_{\mathrm{BHB}}^{\mathrm{RGB}}$ is probably more affected by red leak than the parameter $\Delta U_{\mathrm{TO}}^{\mathrm{RGB}}$.
The obtained results concern metal-poor GCs. Deep UV photometry in metal-rich $\mathrm{GCs},[\mathrm{Fe} / \mathrm{H}]_{Z W}>-1.0$, is needed to investigate the same problems in this range of metallicity. Moreover, strictly speaking, in our sample of GCs observed at NTT (i.e. in $U^{\prime}$ bandpass) there is no GC with sufficient number of stars belonging to the RHB. Thus it is difficult to reach definite conclusions about the apparent position (relative to the TO point and BHB) of the RHB in CMDs with $U$ and $U^{\prime}$ magnitude axes. Also, it is unclear whether (and how exactly) the RHB position in these diagrams depends on metallicity. Our very preliminary conclusion, based on indirect and insufficient evidence is that the position of the RHB, like that of the RGB, does not show a (notable) dependence, as does BHB, on the presence or absence of the Bulmer jump in UV ( $U$ or $\left.U^{\prime}\right)$ bandpass.

Acknowledgements. We thank the anonymous referee for useful comments that have improved the manuscript. We are grateful to Marcio Catelan and Andrzej Udalski for kindly providing data on photometry in M 75 and on the response curve of the $U$ bandpass realized at the $1.3 \mathrm{~m}$ Warsaw telescope. V.K. acknowledges support from the Universidad Católica del Norte through research grant DGIP 10301180.

\section{References}

Alcaíno, G., Liller, W., Alvarado, F., et al. 1996a, AJ, 112, 2004 Alcaíno, G., Liller, W., Alvarado, F., et al. 1996b, AJ, 112, 2020 Alcaíno, G., Liller, W., Alvarado, F., et al. 1997a, AJ, 114, 1067 Alcaíno, G., Liller, W., Alvarado, F., et al. 1997b, AJ, 114, 2638 Alcaíno, G., Liller, W., \& Alvarado, F. 1997c, AJ, 114, 2626

Alcaíno, G., Liller, W., Alvarado, F., et al. 1998a, AJ, 115, 1492

Alcaíno, G., Liller, W., Alvarado, F., et al. 1998b, AJ, 116, 2415

Alcaíno, G., Liller, W., Alvarado, F., et al. 1999, A\&AS, 136, 461 Alvarado, F., Wenderoth, E., Alcaino, G. \& Liller, W. 1990, AJ, 99, 1501 Ažusienis, A., \& Straižys, V. 1966, VilOB, 18, 3

Catelan, M., Borissova, J., Ferraro, F. R., et al. 2002, AJ, 124, 364

Corwin, T. M., Catelan, M., Smith, H. A., et al. 2003, AJ, 125, 2543

Ferraro, F. R., Messineo, M., Fusi Pecci, F., et al. 1999, AJ, 118, 1738

Grebel, E. K., \& Roberts, W. J. 1995, A\&AS, 109, 293

Harris, W. E. 1975, ApJS, 29, 397

Harris, W. E. 1996, AJ, 112, 1487

Holtzman, J. A., Burrows, C. J., Casertano, S., et al. 1995, PASP, 107, 1065

Kravtsov, V. V. 1989, Soviet. Astron. Lett., 15, 356

Kravtsov, V., Ipatov, A., Samus, N., et al. 1997, A\&AS, 125, 1

Momany, Y., Cassisi, S., Piotto, G., et al. 2003, A\&A, 407, 303

Piotto, G., King, I. R., Djorgovski, S. G., et al. 2002, A\&A, 391, 945

Sandquist, E. L., \& Bolte, M. 2004, ApJ, 611, 323

Stetson, P. B. 1987, PASP, 99, 191

Stetson, P. B. 1991, DAOPHOT II Users Manual

Straižys, V. 1973, A\&A, 28, 349

Straižys, V. L. 1977, Multicolor stellar photometry (Vilnus: Mokslas Publishers) 128 (in Russian edition)

Zinn, R., \& West, M. J. 1984, ApJS, 55, 45 